\title{
Functional resistance training can increase strength, knee torque ratio, and functional performance in elderly women
}

\author{
Ahlan Benezar Lima', Ewertton de Souza Bezerra', Lucas Bet da Rosa Orssatto ${ }^{2 *}$, Ericles de Paiva Vieira', \\ Luhan Ammy Andrade Picanço', João Otacilio Libardoni dos Santos'
}

${ }^{1}$ Human Performance Laboratory, Faculty of Physical Education and Physiotherapy, Federal University of Amazonas, Manaus, Brazil

Biomechanics Laboratory, Sports Centre, Federal University of Santa Catarina, Santa Catarina, Brazil

Functional resistance training can increase strength, knee torque ratio, and functional performance in elderly women. The aim of the present study was to understand the effects of closed kinetic chain exercises with constant load on muscular strength, the knee torque conventional ratio (hamstring:quadriceps $-\mathrm{H}: \mathrm{O}$ ), and functional capacity in the elderly. Nine untrained healthy elderly women participated in experimental resistance training. Ten-repetition maximum (10RM) for the deadlift, isokinetic maximum voluntary concentric contraction, and functional capacity were assessed before and after the 7-week resistance-training program. Magnitude based inference analysis was used to examine the differences in muscle strength and functional performance. Therefore, the smallest worthwhile change was calculated and $90 \%$ confidence intervals were also determined to characterize muscle strength and functional performance. The analysis demonstrated an increase in the deadlift and knee flexor torque $(60 \% \mathrm{sec})$ after the experimental intervention. Furthermore, stair ascent, knee extensor torque $(120 \% \mathrm{sec})$, knee flexor torque $(120 \% \mathrm{sec})$, and knee ratio $(60 \% \mathrm{sec})$ also presented a positive effect in the same training period. Conversely, knee extensor torque $(60 \% \mathrm{sec})$, and knee ratio $(120 \% \mathrm{sec})$ did not show conclusive responses. In conclusion, resistance training with functional closed kinetic chain exercises and constant volume load (i.e., $65 \%$ of 1-repetition maximum) can increase deadlift 10RM, isokinetic concentric torque, the conventional $\mathrm{H}: \mathrm{O}$ ratio, and stair ascent performance in elderly women.

Keywords: Aging, Isokinetic testing, Conventional ratio, Strength training

\section{INTRODUCTION}

Aging is associated with the process of reduction in strength and muscle mass (Manini and Clark, 2012). This process has a high impact on the reduction in the capacity to perform daily living activities (e.g., walking, sitting, and stair negotiation) and an increased risk of falls in elderly populations (Scott et al., 2015). Furthermore, women appear to be affected earlier and to a greater magnitude than men (Leyva et al., 2016). Among all strategies for increasing strength and improving functional ability in the elderly, resistance training has been shown to be a safe and effective intervention (Borde et al., 2015; Byrne et al., 2016; Steib et al., 2010). However, resistance training involves a great number of variables, such as: muscle action, rest periods, repetition velocity, frequency, exercise selection and order; and loading and volume (Bird et al., 2005).

In addition, the loading and volume interplay has been reported as a critical factor for positive effects on strength gains in different populations (Fisher et al., 2013; Fisher et al., 2017; Schoenfeld, 2013), as well as which loading and volume can determine the optimal range of training. In particular the loading and volume interplay is based on the training principle of progression; it indicates the necessity to change the load volume in resistance training programme designs (Bird et al., 2005).

For improvements in muscular strength in older subjects, the American College of Sports Medicine recommends the use of in-

\footnotetext{
${ }^{*}$ Corresponding author: Lucas Bet da Rosa Orssatto (iD) https://orcid.org/0000-0003-3788-3700

Laboratório de Biomecânica, Centro de Desportos, Universidade Federal de Santa Catarina, Campus Reitor João David Ferreira Lima, Trindade, Florianópolis, Santa Catarina CEP 88040-900, Brazil

Tel: +5548988092900, Fax: +554837218530, E-mail: lucasorssatto@gmail.com

Received: May 6, 2018 / Accepted: June 14, 2018
} 
tensities between $60 \%-80 \%$ of one-repetition maximum (1RM) and moderate to slow contraction velocity (Ratamess et al., 2009). Moreover, a recent meta-analysis (Borde et al., 2015; Steib et al., 2010) suggests that higher intensities result in greater improvements in strength compared to moderate intensities. However, the use of greater intensities in untrained elderly individuals requires longer periods of recovery for neuromuscular (i.e., explosive force, power, and strength) resistance training-related acute impairments, exposing the elderly to reduced balance and functional capacity and an increased risk of falling (Orssatto et al., 2018a). Following this rationale, lower intensities (i.e., $60 \%$ of 1RM) would be safer and efficient for strength increases for untrained elderly, while the intensity could be gradually increased in subsequent months of training.

Conversely, the elderly require increases in functional capacity, while Walker et al. (2017) suggested that training intensities of -50\%-60\% and slow contraction velocities did not provide improvements in functional capacity. However, it can be hypothesized that these results could be explained by the exercise choice, and not by the adopted intensity. The cited study adopted nonfunctional exercises for lower limbs (i.e., horizontal leg press, knee extension, and knee flexion), which do not follow the movement pattern specificity of daily living activities. In this context, the choice of multi-articular and closed kinetic chain functional exercises, such as the squat or deadlift (more related to daily living movements), would result in improvements in functional capacity beyond strength in the elderly. Despite these exercises being considered functional and involving activation of knee extensors and flexors (Escamilla et al., 2001), it is unclear in the literature if the increases in strength between knee antagonist muscles is similar. For example, recently Orssatto et al. (2018b) reported increases in knee extension but not knee flexion torque that consequently reduced knee torque ratio, after 12 weeks of resistance training using the leg press, which is characterized by participation of both knee extensors and knee flexors (Walker et al., 2011).

Thus, the aim of the present study was to understand the effects of closed kinetic chain exercises with constant load on muscular strength, the knee torque conventional ratio, and functional capacity in the elderly.

\section{MATERIALS AND METHODS}

\section{Experimental design}

The study design was a pre-post nonrandomized design. During the first visit, subjects were informed about the study de- sign, procedures, and performed body mass and height assessments. On the subsequent three days, each one separated by 48 $\mathrm{hr}$, subjects performed familiarization sessions. $48 \mathrm{hr}$ after the familiarization period, the subjects attended two visits, each separated by a minimum of $48 \mathrm{hr}$, during which they were tested and retested for baseline values in: (a) Isokinetic maximum voluntary concentric contraction, (b) functional capacity, and (c) ten-repetitions maximum (10RM). After testing, subjects started the resistance training program. Thereafter (posttraining), each subject completed the same tests performed at baseline.

\section{Subjects}

Subjects completed a health history and physical activity questionnaire. Participants were required to meet the following inclusion criteria: $\geq 60$ years old, physically independent, free from cardiac disease, free from orthopaedic dysfunction, and not having performed noncontrolled physical activity within one year preceding the beginning of the study. Fourteen women were initially eligible; however, after the intervention period only nine subjects $(70.7 \pm 6.5 \mathrm{~kg} ; 1.53 \pm 0.03 \mathrm{~m} ; 64.1 \pm 2.1$ years) completed all stages of the experiment (i.e., five subjects dropped out). One subject withdrew claiming muscular discomfort during the resistance training regime and four did not reach the established minimum session attendance (frequency $>85 \%$ [Gentil and Bottaro, 2013]). Adhesion to the program was satisfactory, with all subjects participating in $\geq 13$ (>90\%) of the total sessions. Written informed consent was obtained from all subjects after providing a detailed description of study procedures. All procedures performed in this study were approved by a local Institutional Ethics Committee (approval number: 1.753.546) and followed the ethical guidelines of the Declaration of Helsinki (64th World Medical Association General Assembly, Fortaleza, Brazil, October 2013).

\section{Physical performance tests}

Subjects performed a 10RM test in the deadlift exercise. Tests' quality control was adapted from Orssatto et al. (2018a) study procedures. Knee joint range of motion was standardized from $\sim 90^{\circ}$ to $\sim 5^{\circ}$ of flexion during the deadlift measured by a goniometer. Using the same equipment and settings, the same evaluator conducted the familiarization, pre- and posttests. To ensure that the true 10RM was reached, subjects were encouraged to perform an eleventh repetition after the tenth. Thus, if less than 10 repetitions were performed, the weight was reduced for the next trial, and if eleven repetitions were performed, the weight was increased. The 10RM test was considered successful when the sub- 
jects performed exactly 10 repetitions. Each subject performed 1-3 trials with a rest period of $5 \mathrm{~min}$. All subjects performed a specific leg press warm up ( $50 \%$ load performed in the final familiarization session) before starting the 10RM test.

Concentric knee extensor and flexor peak torques of the preferred limb were assessed on an isokinetic dynamometer (Biodex System 4 Pro, Biodex Medical Systems, Shirley, NY, USA). The subjects were seated at $70^{\circ}$ and $90^{\circ}$ for hip and knee angle joints, respectively $\left(0^{\circ} 1 / 4\right.$ knee fully extend). Subjects were verbally encouraged to perform three maximum concentric knee extensor $\left(\mathrm{Q}_{\mathrm{CON}}\right)$ and flexor $\left(\mathrm{H}_{\mathrm{CON}}\right)$ contractions at $60 \% \mathrm{sec}$ and $120 \% \mathrm{sec}$ isokinetic velocity. A 3-min rest period was allowed between trials. The highest torque value for each test was used for data analysis. Stair ascent was assessed according to Rikli and Jones (2013). Each subject performed two attempts at each test and the fastest time was used for further analyses.

\section{Resistance training program}

At the beginning of each training session, subjects performed a general warm-up routine (treadmill walking [10 min], core training [2 sets of traditional prone plank and supine bridge exercises $\{15$-sec isometric contraction per set $\}$ and one set with $50 \%$ of workout load for the first lower limb exercise]). Next, they performed the deadlift and free squat in all training sessions; the order of these exercises was alternated. The training intervention was completed with the same load (65\% of estimated 1RM [Brzycki, 1993]) and 3 sets of 8-10 repetitions with a rest interval of 2 min between sets. The intervention protocol included 14 sessions (two sessions per week). The cadence of exercise was $2 \mathrm{sec}$ for both the concentric and eccentric phases. Following the performance of lower limb training, a further complementary upper-body program was performed, consisting of 3 sets of $8-10$ repetitions of the bench press and anterior lat pull down with a 2-min rest between sets. During all sessions, subjects were directly supervised

Table 1. Data reliability

\begin{tabular}{|c|c|c|c|c|}
\hline \multirow{2}{*}{ Variable } & \multicolumn{2}{|c|}{ Pre } & \multicolumn{2}{|c|}{ Post } \\
\hline & ICC & $\% C V$ & ICC & $\% C V$ \\
\hline Stair ascent & 0.84 & 7.2 & 0.89 & 3.8 \\
\hline $\mathrm{O}_{\mathrm{con}} 60^{\circ} / \mathrm{sec}$ & 0.99 & 3.3 & 0.99 & 1.7 \\
\hline $\mathrm{H}_{\mathrm{con}} 60^{\circ} / \mathrm{sec}$ & 0.98 & 4.8 & 0.99 & 1.5 \\
\hline $0_{\text {con }} 120^{\circ} / \mathrm{sec}$ & 0.99 & 3.4 & 0.99 & 1.7 \\
\hline $\mathrm{H}_{\mathrm{con}} 120^{\circ} / \mathrm{sec}$ & 0.99 & 2.2 & 0.95 & 3.2 \\
\hline
\end{tabular}

$\mathrm{O}_{\text {con, }}$ concentric knee extensor; $\mathrm{H}_{\mathrm{coN}}$, concentric knee flexor; ICC, intraclass correlation coefficient; $\mathrm{CV}$, coefficient of variation. (i.e., strength and conditioning trainers) to help ensure consistent and safe performance.

\section{Statistical analyses}

Magnitude based inference analysis was used to examine the differences in muscle strength and functional performance using a customized spreadsheet (Hopkins et al., 2009). The smallest worthwhile change was calculated (i.e., $0.2 \times$ by the between-subjects standard deviation [SD]) and $90 \%$ confidence intervals were determined to characterize muscle strength and functional performance (Hopkins et al., 2009). The quantitative chances of higher, similar, or lower differences were evaluated qualitatively as follows: $<1 \%$, almost certainly not; $1 \%$ to $5 \%$, very unlikely; $5 \%$ to $25 \%$, unlikely; $25 \%$ to $75 \%$, possible; $75 \%$ to $95 \%$, likely; $95 \%$ to $99 \%$, very likely; >99\%, almost certain. The true difference was assessed as unclear when the chances of having positive and negative results were both $>5 \%$. The threshold values for Cohen effect size (ES) statistics were $>0.2$ (small), $>0.5$ (moderate), and $>0.8$ (large). However, only differences classified as equal/higher likely and moderate ES were used as criteria for describing changes between groups. Values are reported as mean values \pm and $\mathrm{SD}$ unless otherwise stated. Data reliability was calculated with the intraclass correlation coefficient and technical error $(\% \mathrm{CV})$ for the isokinetic and functional pre and post variables (Hopkins, 2000) (Table 1).

\section{RESULTS}

The analysis demonstrated an increase in the deadlift and knee flexor torque $\left(60^{\circ} / \mathrm{sec}\right)$ after the experimental intervention. Furthermore, stair ascent, knee extensor torque $(120 \%$ sec), knee flexor

Table 2. Pre and post mean \pm standard deviation, percentage changes $(\Delta \%)$ and effect size (ES) for the assessed variables.

\begin{tabular}{|c|c|c|c|c|}
\hline Variable & Pre & Post & $\Delta \%$ & ES \\
\hline Deadlift (kg) & $25.3 \pm 3.2$ & $29.1 \pm 2.7$ & $17.2 \pm 22.2$ & 1.08 \\
\hline Stair ascent (sec) & $3.9 \pm 0.4$ & $3.7 \pm 0.3$ & $-5.5 \pm 10.6$ & 0.50 \\
\hline $\mathrm{O}_{\operatorname{con}} 60^{\circ} / \sec (\mathrm{N} \cdot \mathrm{m})$ & $98.9 \pm 17.5$ & $105.2 \pm 10.6$ & $9.2 \pm 22.3$ & 0.32 \\
\hline $\mathrm{H}_{\text {con }} 60^{\circ} / \mathrm{sec}(\mathrm{N} \cdot \mathrm{m})$ & $43.5 \pm 7.1$ & $50.9 \pm 6.7$ & $19.1 \pm 20.2$ & 0.94 \\
\hline $\mathrm{O}_{\mathrm{CON}} 120^{\circ} / \mathrm{sec}(\mathrm{N} \cdot \mathrm{m})$ & $78.1 \pm 14.1$ & $86.1 \pm 8.4$ & $14.4 \pm 29.0$ & 0.51 \\
\hline $\mathrm{H}_{\text {con }} 120^{\circ} / \mathrm{sec}(\mathrm{N} \cdot \mathrm{m})$ & $41.6 \pm 10.0$ & $47.6 \pm 4.6$ & $20.1 \pm 29.0$ & 0.54 \\
\hline $\mathrm{H}: 0$ ratio $\left(60^{\circ} / \mathrm{sec}\right)$ & $0.44 \pm 0.07$ & $0.49 \pm 0.06$ & $11.3 \pm 21.8$ & 0.58 \\
\hline $\mathrm{H}: \mathrm{Q}$ ratio $\left(120^{\circ} / \mathrm{sec}\right)$ & $0.54 \pm 0.10$ & $0.56 \pm 0.06$ & $8.0 \pm 29.6$ & 0.16 \\
\hline
\end{tabular}

Values are presented as mean \pm standard deviation.

$\mathrm{O}_{\text {con, }}$ concentric knee extensor; $\mathrm{H}_{\text {con, }}$ concentric knee flexor; $\mathrm{H}: \mathrm{Q}$, hamstring:quadriceps. 


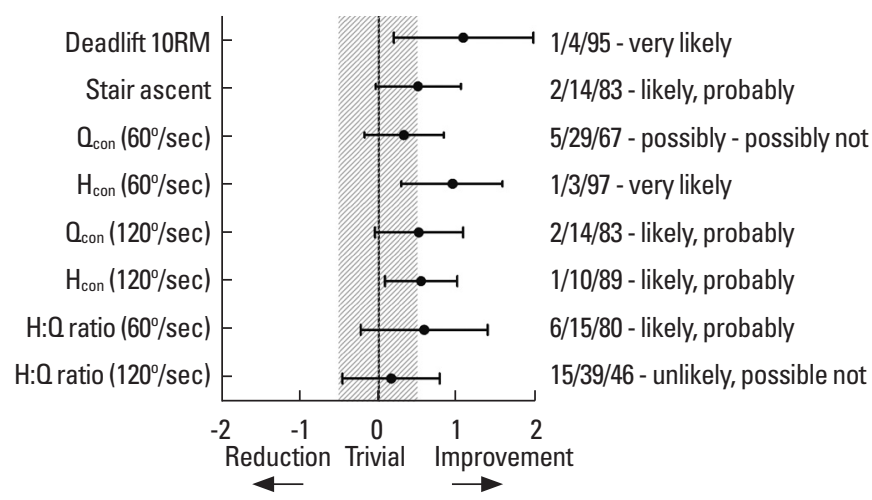

Fig. 1. Magnitude based inferences for improvement (positive), reduction (negative), or inconclusive (trivial) in all experimental measures. Data are reported as (mean [post-pre] $\pm 90 \%$ confidence limits). The smallest worthwhile change trivial zone was set at $20 \%$ of the pretraining standard deviation for each variable. 10RM, ten-repetition maximum; $\mathrm{H}: \mathrm{O}$, hamstring:quadriceps; $\mathrm{Q}_{\text {con, }}$ concentric knee extensor torque; $\mathrm{H}_{\mathrm{coN}}$, concentric knee flexor torque.

torque $\left(120^{\circ} / \mathrm{sec}\right)$, and knee ratio $\left(60^{\circ} / \mathrm{sec}\right)$ also presented a positive effect in the same training period. Conversely, knee extensor torque $\left(60^{\circ} / \mathrm{sec}\right)$ and knee ratio $\left(120^{\circ} / \mathrm{sec}\right)$ did not show conclusive responses (Table 2, Fig. 1).

\section{DISCUSSION}

The main findings in the present study suggest that the use of the deadlift and squat as functional exercises with constant load can improve muscular strength, knee conventional ratios, and functional capacity in elderly women. These results have relevant practical applications regarding exercise and training intensity prescription in this population.

The improvements in deadlift 10RM observed in the present study ( 1.9\% per week) are in agreement with several studies that adopted similar training intensities. For example, Walker et al. (2017) and Tsutsumi et al. (1998), respectively reported increases in leg press $1 \mathrm{RM}(+12 \%, \sim 1 \%$ per week $)$ and knee extension $1 \mathrm{RM}(+36 \%, \sim 3 \%$ per week) with an intensity of $\sim 50 \%-$ $65 \%$ of $1 \mathrm{RM}$, after 12 weeks of strength training. Additionally, Vincent et al. (2002) reported increases in leg press 1RM after 24 weeks $(+17 \%, \sim 0.7 \%$ per week) with an intensity of $50 \%$ of 1RM.

Although a meta-analysis suggested that higher training intensities result in greater improvements in strength in the elderly, untrained elders are subject to acute neuromuscular impairments following resistance training, wherein higher intensities require longer recovery periods (Steib et al., 2010). During this recovery, the elderly seem to experience reduced ability to recover maxi- mum torque, explosive torque, and power (Orssatto et al., 2018a) which are related to static as well dynamic balance (Izquierdo et al., 1999), thus increasing the risk of falling (Bento et al., 2010; Ema et al., 2016). Therefore, it would be safer to start the training program with intensities near $60 \%$ of $1 \mathrm{RM}$ and then increase the intensity gradually in subsequent months. The present study design resulted in chronic improvements in strength after 7 weeks of strength training without exposing the subjects to an increased risk of falling. Indeed, to ensure continuous improvements in strength it is necessary to increase intensity in subsequent weeks, when the repeated bout effect would protect the subjects and help to avoid exacerbated neuromuscular impairment following resistance training (Hyldahl et al., 2017; Orssatto et al., 2018a).

Another strength parameter assessed in the present study, the isokinetic concentric strength altered $\left(\mathrm{Q}_{\mathrm{CON}}, 60 \% \mathrm{sec}=+0.9 \%\right.$ and $120 \% \mathrm{sec}=+1.4 \%$; and $\mathrm{H}_{\mathrm{CON}}, 60 \% \mathrm{sec}=+2.4 \%$ and $120 \% \mathrm{sec}=+2 \%$, per week) and probably as a consequence the $\mathrm{H}: \mathrm{Q}$ ratio $\left(60^{\circ} / \mathrm{sec}\right)$ also changed (moderate ES). Our outcomes corroborate with other resistance training studies in the elderly. Orssatto et al. (2018b) reported significant increases of $\sim 20 \%$ in $\mathrm{Q}_{\mathrm{CON}}(60 \% \mathrm{sec})$ peak torque $(\sim 1.7 \%$ per week) and nonsignificant increases of $\sim 18 \%$ in $\mathrm{H}_{\text {CON }}(60 \%$ sec) peak torque (1.5\% per week). One potential explanation for positive adaptation during constant volume load is that the deadlift and squat show high muscle activity in the biceps femoris (55\%) and vastus lateralis (91\%) during the maximum voluntary isometric contraction, respectively (Ebben et al., 2009), and these exercises were included in our resistance training programme with the same volume load.

Improvement in functional task performance has an important influence on the quality of life and reduces the risk of falling in the elderly (World Health Organization, 2007, 2015). Our result regarding stair ascent presented a positive response (moderate ES) even with no change in volume load. The exercises selected during the resistance training programme were the deadlift and squat, both of which require vertical centre of gravity displacement during the carrying of the load, and a similar displacement can be observed during stair ascent. Conversely, Walker et al. (2017) reported no statistical improvements in stair ascent after 12 weeks of resistance training using nonfunctional exercises (i.e., leg press, knee extension, and knee flexion exercises) and a similar intensity to that adopted in the present study ( $50 \%-60 \%$ of $1 \mathrm{RM})$. One indicative that exercise choice might play a role in functional capacity adaptations, is that MAT Pilates can improve stair ascent performance of elderly women after 6 weeks of training (Bertoli et al., 2017). 
Strengths and limitations should be reported. Using the same experienced evaluator to perform all tests gives our study high internal validity. Another strength of the study is that we used a homogenous population of elderly unaccustomed to resistance training. On the other hand, the absence of a control group in the experimental design protocol does not enable an exact response. Furthermore, the results from the present study cannot be extrapolated to previously trained, older (e.g., $>80$ years), or frail subjects. Further studies should be developed with a larger sample size to confirm our findings.

As a practical application for resistance training prescription, trainers should consider the lack of necessity to adjust the intensity in the first weeks of resistance training for untrained elderly people, since this training includes closed kinetic chain exercises such as the squat and deadlift. Thereafter, training intensity can be increased gradually in subsequent weeks to ensure safety for daily living activities (Orssatto et al., 2018a). In conclusion, resistance training with functional closed kinetic chain exercises and constant volume load (i.e., $65 \%$ of $1 \mathrm{RM}$ ) can increase deadlift 10RM, isokinetic concentric torque, the conventional H:Q ratio, and stair ascent performance of elderly women.

\section{CONFLICT OF INTEREST}

No potential conflict of interest relevant to this article was reported.

\section{ACKNOWLEDGMENTS}

JOLS would like to thank the National Council for Scientific and Technological Development (CNPq) for the research fellowships to $\mathrm{AB}$ and $\mathrm{EV}$, and MSc scholarship for LA. Furthermore, ESB would like to thank the Foundation for Research Support of the State of Amazonas (FAPEAM) for conceding a PhD scholarship.

\section{REFERENCES}

Bento PC, Pereira G, Ugrinowitsch C, Rodacki AL. Peak torque and rate of torque development in elderly with and without fall history. Clin Biomech (Bristol, Avon) 2010;25:450-454.

Bertoli J, Biduski GM, de la Rocha Freitas C. Six weeks of Mat Pilates training are enough to improve functional capacity in elderly women. J Bodyw Mov Ther 2017;21:1003-1008.

Bird SP, Tarpenning KM, Marino FE. Designing resistance training pro- grammes to enhance muscular fitness: a review of the acute programme variables. Sports Med 2005;35:841-851.

Borde R, Hortobágyi T, Granacher U. Dose-response relationships of resistance training in healthy old adults: a systematic review and meta-analysis. Sports Med 2015;45:1693-1720.

Brzycki M. Strength testing-predicting a one-rep max from reps-to-fatigue. J Phys Educ Recreat Danc 1993;64:88-90.

Byrne C, Faure C, Keene DJ, Lamb SE. Ageing, Muscle power and physical function: a systematic review and implications for pragmatic training interventions. Sports Med 2016;46:1311-1332.

Ebben WP, Feldmann CR, Dayne A, Mitsche D, Alexander P, Knetzger KJ. Muscle activation during lower body resistance training. Int J Sports Med 2009;30:1-8.

Ema R, Saito M, Ohki S, Takayama H, Yamada Y, Akagi R. Association between rapid force production by the plantar flexors and balance performance in elderly men and women. Age (Dordr) 2016;38:475483.

Escamilla RF, Fleisig GS, Zheng N, Lander JE, Barrentine SW, Andrews JR, Bergemann BW, Moorman CT 3rd. Effects of technique variations on knee biomechanics during the squat and leg press. Med Sci Sports Exerc 2001;33:1552-1566.

Fisher J, Steele J, Bruce-Low S, Smith D. Evidence-based resistance training recommendations for muscular hypertrophy. Med Sport 2013;17: 217-235.

Fisher J, Steele J, Smith D. High- and low-load resistance training: interpretation and practical application of current research findings. Sports Med 2017;47:393-400.

Gentil P, Bottaro M. Effects of training attendance on muscle strength of young men after 11 weeks of resistance training. Asian J Sports Med 2013;4:101-106.

Hopkins WG. Measures of reliability in sports medicine and science. Sports Med 2000;30:1-15.

Hopkins WG, Marshall SW, Batterham AM, Hanin J. Progressive statistics for studies in sports medicine and exercise science. Med Sci Sports Exerc 2009;41:3-13.

Hyldahl RD, Chen TC, Nosaka K. Mechanisms and mediators of the skeletal muscle repeated bout effect. Exerc Sport Sci Rev 2017;45:24-33.

Izquierdo M, Aguado X, Gonzalez R, López JL, Häkkinen K. Maximal and explosive force production capacity and balance performance in men of different ages. Eur J Appl Physiol Occup Physiol 1999;79:260267.

Leyva A, Balachandran A, Signorile JF. Lower-body torque and power declines across six decades in three hundred fifty-seven men and women: a cross-sectional study with normative values. J Strength Cond Res 2016;30:141-158. 
Manini TM, Clark BC. Dynapenia and aging: an update. J Gerontol A Biol Sci Med Sci 2012;67:28-40.

Orssatto LB, Moura BM, Bezerra ES, Andersen LL, Oliveira SN, Diefenthaeler F. Influence of strength training intensity on subsequent recovery in elderly. Exp Gerontol 2018a;106:232-239.

Orssatto LB, Moura BM, Sakugawa RL, Radaelli R, Diefenthaeler F. Leg press exercise can reduce functional hamstring:quadriceps ratio in the elderly. J Bodyw Mov Ther 2018b;22:592-597.

Ratamess NA, Alvar BA, Evetoch TK, Housh TJ, Kibler WB, Kraemer WJ, Triplett NT. Progression models in resistance training for healthy adults. Med Sci Sports Exerc 2009;41:687-708.

Rikli RE, Jones CJ. Development and validation of criterion-referenced clinically relevant fitness standards for maintaining physical independence in later years. Gerontologist 2013;53:255-267.

Schoenfeld BJ. Is there a minimum intensity threshold for resistance training-induced hypertrophic adaptations? Sports Med 2013;43:1279-1288.

Scott D, Daly RM, Sanders KM, Ebeling PR. Fall and fracture risk in sarcopenia and dynapenia with and without obesity: the role of lifestyle interventions. Curr Osteoporos Rep 2015;13:235-244.

Steib S, Schoene D, Pfeifer K. Dose-response relationship of resistance training in older adults: a meta-analysis. Med Sci Sports Exerc 2010; 42:902-914.

Tsutsumi T, Don BM, Zaichkowsky LD, Takenaka K, Oka K, Ohno T. Comparison of high and moderate intensity of strength training on mood and anxiety in older adults. Percept Mot Skills 1998;87(3 Pt 1): 1003-1011.

Vincent KR, Braith RW, Feldman RA, Magyari PM, Cutler RB, Persin SA, Lennon SL, Gabr AH, Lowenthal DT. Resistance exercise and physical performance in adults aged 60 to 83. J Am Geriatr Soc 2002;50:11001107.

Walker S, Haff GG, Häkkinen K, Newton RU. Moderate-load muscular endurance strength training did not improve peak power or functional capacity in older men and women. Front Physiol 2017;8:743.

Walker S, Peltonen H, Avela J, Häkkinen K. Kinetic and electromyographic analysis of single repetition constant and variable resistance leg press actions. J Electromyogr Kinesiol 2011;21:262-269.

World Health Organization. Global report on falls prevention in older age. Geneva (Switzerland): World Health Organization; 2007.

World Health Organization. World report on ageing and health. Geneva (Switzerland): World Health Organization; 2015. 